Incidencia de la comunicación
Incidencia de la comunicación | Revista Virtual

Universidad Católica del Norte, 63, 94-125

ISSN: 0124-5821 (En línea)

Jiménez Peñarreta, K. M., Sánchez Montalván, S. E., y Rodríguez Recalde, J. S. (mayoagosto, 2021). Incidencia de la comunicación interna en la identidad institucional del docente de la Universidad Politécnica Salesiana. Revista Virtual Universidad Católica del Norte, (63), 94-

125. https://www.doi.org/10.35575/rvucn.n63a5

\title{
Incidencia de la comunicación interna en la identidad institucional del docente de la Universidad Politécnica Salesiana
}

Incidence of internal communication on the institutional identity of Universidad Politécnica

Salesiana's teachers

Katherine Mishel Jiménez Peñarreta

Ingeniera en Gerencia y Liderazgo

Carrera de Gerencia y Liderazgo, Universidad Politécnica Salesiana

Quito, Ecuador

kjimenezp@est.ups.edu.ec

Orcid: https://orcid.org/0000-0002-6183-8494

\section{Soraya Elizabeth Sánchez Montalván}

Doctora en Ciencias Administrativas

Carrera de Gerencia y Liderazgo, Universidad Politécnica Salesiana

Quito, Ecuador

ssanchez@ups.edu.ec

Orcid: https://orcid.org/0000-0002-2875-7174

Juan Sebastián Rodríguez Recalde

Ingeniero en Gerencia y Liderazgo

Carrera de Gerencia y Liderazgo, Universidad Politécnica Salesiana

Quito, Ecuador

juanseb_0603@hotmail.com

Orcid: https://orcid.org/0000-0002-5343-123X

Recibido: 05 de julio de 2020

Evaluado: 15 de septiembre de 2020

Aprobado: 26 de febrero de 2021

Tipo de artículo: Investigación Científica y Tecnológica 
Incidencia de la

comunicación
Incidencia de la comunicación | Revista Virtual

Universidad Católica del Norte, 63, 94-125

ISSN: 0124-5821 (En línea)

\title{
Resumen
}

La presente investigación es una construcción académica que correlaciona la percepción identitaria del docente con la trasmisión de contenidos, desde la dirección de la Universidad Politécnica Salesiana; planteándose como objetivo general, identificar la incidencia de la comunicación interna en la identidad institucional del docente de esta universidad, en el año 2019. Para establecerlo se utilizó una metodología mixta (cuali-cuantitativa) que integra las técnicas de análisis del discurso, de comunicaciones emitidas por la dirección de la universidad, entrevistas semiestructuradas y la aplicación de encuestas, a una muestra probabilística estratificada de 515 docentes de todas sus sedes en Quito, Guayaquil y Cuenca. El estudio mostró que el docente se siente identificado con la filosofía y cultura organizacional, lo que conllevó a inferir que ciertamente asume, de forma activa, la identidad institucional motivada por la comunicación; aunque se revelen también ciertos déficits comunicativos. Consecuentemente, esta investigación complementó los estudios cuantitativos y cualitativos de gestión de identidad y comunicación institucional en la educación superior; y, en el sujeto de estudio, aportó a la consolidación del Modelo Educativo Salesiano que propone una educación integral, sostenida por la filosofía institucional en el accionar del docente, dentro de la formación académica, investigación y vinculación con la sociedad.

Palabras clave: Comunicación interna; Cultura organizacional; Docente; Filosofía organizacional; Identidad institucional; Identidad organizacional; Universidad.

\begin{abstract}
The present study is an academic construction that correlates the teachers' identity perception with the transmission of content from the Directorate of Universidad Politécnica Salesiana, with the general objective of identifying the incidence of internal communication on the institutional identity of the teachers at this university in 2019. To establish it, a mixed (qualitative quantitative) methodology is used. This integrates the techniques of analysis of the communication discourse issued by the University Directorate, semi-structured interviews, and the application of surveys to a stratified probabilistic sample of 515 teachers from all its headquarters in Quito, Cuenca, and Guayaquil. This study showed that the teachers feel identified with the organizational
\end{abstract}


Incidencia de la comunicación
Incidencia de la comunicación | Revista Virtual

Universidad Católica del Norte, 63, 94-125

ISSN: 0124-5821 (En línea)

philosophy and culture, which leaded to infer that they certainly assume, actively, the institutional identity, motivated by communication, although a few communication deficits have also been reported. In this sense, the research complemented the quantitative and qualitative studies on identity management and institutional communication in higher education; and in the subject of study, it contributed to the consolidation of the Salesian Education Model, which proposes comprehensive education sustained by the teachers' actions, within the framework of academic training, research and connection with society.

Keywords: Internal communication; Organizational culture; Teacher; Organizational philosophy; Institutional identity; Organizational identity; University.

\section{Introducción}

Los constantes cambios en la sociedad, la globalización, la competitividad, el gran desarrollo de los mercados, la búsqueda constante de la calidad de los bienes y servicios que se ofrecen, son algunos de los grandes desafíos que deben encarar las instituciones de educación superior, orientando la visión organizacional de estas casas de estudios superiores, no sólo a la formación y preparación de los profesionales, ofreciendo un servicio educativo de calidad, sino, insertar en sus principios básicos la comunicación, la cultura organizacional y la identidad corporativa como ejes de acción, ya que estos tres elementos constituyen de relevancia estratégica como instrumentos de gestión de todos los procesos dinámicos de una institución moderna. (Perozo de Jiménez, 2006, p. 364)

Siguiendo este preludio, que considera la relevancia estratégica de la comunicación e identidad organizacional, en el presente estudio se pretende fortalecer estos ejes claves, identificando si la comunicación, desde la dirección general de la Universidad Politécnica Salesiana, incide o no en la identidad institucional del docente, debido a que, por medio de la información trasmitida, la institución de educación superior comunica su misión, visión, el modelo que determina su identidad y la cultura organizacional (Cea Esteruelas, 2018, p. 101). Partiendo, además, de que la comunicación no únicamente nace de actividades académicas y administrativas que ejecuta la institución de educación superior en estudio, sino también del trabajo que se realiza 
para retomar los mensajes olvidados o para consolidar los aún preservados, que afirman la identidad institucional y la consecuente imagen pública (Pérez y Rodríguez del Bosque, 2014, p. 100); se plantea, entonces, como hipótesis de partida que: "La comunicación interna incide en la identidad institucional del docente de la Universidad Politécnica Salesiana"; y consecuentemente se determinan los siguientes objetivos específicos: identificar las políticas y estrategias de comunicación interna, como parte del proceso de gestión de la identidad institucional, que integre mensajes y simbolismos comunes; contrastar las concepciones sobre la identidad institucional comunicada, en cuanto a creencias, valores, prácticas y filosofía organizacional, presentes en los docentes de la Universidad Politécnica Salesiana; y categorizar los resultados de la investigación de campo para una comprensión de la relación comunicación - identidad. En este contexto, el objetivo general del estudio es identificar la incidencia de la comunicación interna en la identidad institucional del docente de la Universidad Politécnica Salesiana, en el año 2019; diagnóstico que determina si las características de la filosofía y la cultura organizacional respaldan la actual identidad institucional, y la manera como esta se relaciona con la comunicación emitida.

Pineda de Alcázar (2001) enmarca las corrientes teóricas clásicas de la comunicación en norteamericanas, europeas y latinoamericanas. La escuela norteamericana, pionera de la matriz teórica neopositivista, significó un progreso del conocimiento empírico, comercial y administrativo en la comunicación y sus efectos; sin embargo, no estableció una ruptura del paradigma clásico que instituyó Laswell, manteniendo a lo largo de los años una visión funcionalista e instrumental del proceso comunicativo. Por su parte, la teoría europea contempla a la escuela de Frankfurt, el estructuralismo francés y a la escuela Inglesa. La primera en mención, a partir de la visión del psicoanálisis, el marxismo y la sociología crítica, inicia el estudio de la cultura de masas, empero, en los años noventa, ha sido objeto de verificación, en vista de los nuevos cambios en el campo de la comunicación y cultura, dado que se cree que sus propuestas iniciales tienden a una visión pesimista sobre la sociedad de masas y una concepción aristocrática de la cultura. En otro orden, está el estructuralismo francés, con sus análisis sobre los discursos y los textos; esta corriente introduce en el campo de la comunicación los aportes de antropología cultural de Levy Strauss, de la linguística de Ferdinand Saussure y de Roman Jackobson. Posteriormente, la Escuela Inglesa o de los Estudios Culturales orientó sus análisis en los problemas concretos de la cultura contemporánea, su institucionalización y la relación de la cultura 
popular con la masiva, y recalcaron en la posibilidad de un contrapoder practicado por las clases subalternas, introduciendo el concepto de sujeto en la teoría de la comunicación. Finalmente, la corriente teórica latinoamericana instauró una visión propia sobre comunicación alternativa y comunicación humana, entendida como diálogo; esta tendencia que se debe teóricamente a los estudios culturales ingleses y a la escuela de Frankfurt, inició una honda revisión de los movimientos estructuralistas, semiológicos, marxistas y funcionalistas; adicional a eso, estableció una teoría de la comunicación que otorga preminencia al campo del lenguaje, adjudicando un giro en los estudios de audiencia, desde perspectivas antropológicas, etnográficas, sociológicas e históricas, llenando así vacíos teóricos en las ciencias de la comunicación.

Con base en estos enfoques, se enmarca este estudio en la corriente estructuralista, acogiendo como disciplinas a la pragmática y la semiótica que en los últimos años han enriquecido los estudios referentes al discurso y los textos; adoptando así como metodología de esta investigación el descubrimiento de conceptos, categorías y patrones, expuestos en el análisis de discurso y contenidos; en este eje, Van Dijk (2001) establece que dentro de la teoría semiótica el título utilizado para expresar el objeto específico de este pensamiento es el concepto de "interpretación" como proceso de atribución; es decir, la interpretación de un discurso es la asignación de significados a los contenidos. Por la misma línea, las condiciones de los actos de habla en el discurso, relativos a contextos pragmáticos, son comúnmente formuladas en expresiones de preferencias, conocimientos y creencias. Estas condiciones son abstracciones del contexto comunicativo: cómo el emisor y receptor planea, ejecuta, comprende, memoriza, acepta $\mathrm{y}$, en general, modifica su juicio respecto a los actos de habla; estos tópicos demandan de un modelo que dé cuenta de la forma en que los usuarios individuales del lenguaje conducen los actos de habla en un contexto.

\section{Comunicación}

La comunicación es un elemento esencial en el funcionamiento de cualquier organización; sus flujos conectan a la empresa como un sistema que integra un todo (Eltanskaya et al., 2019, p. 1). Para Schoeneborn et al. (2019) la comunicación es el medio por el cual la información es transmitida y compartida a un conjunto de personas (p. 476). Por su parte, Stolyarchuk et al. (2019) 
Incidencia de la

comunicación
Incidencia de la comunicación | Revista Virtual

Universidad Católica del Norte, 63, 94-125

ISSN: 0124-5821 (En línea)

refieren que la comunicación es la trasmisión de información entre individuos, en forma de mensajes orales o escritos (p. 2).

En este sentido, la comunicación debe integrar la transmisión y la compresión del mensaje. Comunicar va más allá de emitir mensajes, ese mensaje también es importante que se entienda (Robbins y Judge, 2017, p. 345).

Comunicar y realizar seguimiento son dos elementos que van intrínsecamente ligados; debido a que, para alcanzar una comunicación eficaz, se torna importante disponer de herramientas que permitan determinar si esa comunicación funciona o no, aporta o no, es comprensible o no (Garnica Gómez, 2011).

La comunicación puede fluir de forma vertical o lateral. La dimensión vertical se subdivide asimismo en las direcciones ascendente y descendente. La comunicación que fluye desde el nivel de un grupo u organización hasta un nivel inferior se denomina comunicación descendente. La utilizan los líderes y gerentes de grupos para asignar metas, dar instrucciones sobre el trabajo, explicar las políticas y los procedimientos, señalar los problemas que necesitan atención y brindar retroalimentación acerca del desempeño. (Robbins y Judge, 2017, p. 347)

De forma complementaria, la comunicación interna contribuye a generar un gran compromiso de los colaboradores, ya que potencia mayores dinámicas relacionales, participativas y de sentimientos de pertenencia, basados en la valorización de su capital humano y en una efectiva gestión de interacción con sus miembros (Brandão, 2018, p. 96).

Asimismo, Abadía Sánchez y Vaca López (2012) asumen que lo que persigue la comunicación organizacional es el soporte para alcanzar los objetivos, sin tomar en cuenta el tipo de organización, puesto que la comunicación, al irse generando en el diario vivir, se transforma en un arduo proceso que implica "la convergencia de los lenguajes, los imaginarios, las narrativas, las percepciones y demás fenómenos comunicacionales" (Abadía Sánchez y Vaca López, 2012, p. 39).

En este proceso, el capital humano es un actor fundamental debido al grado de responsabilidad con el que cuenta, al tener que saber qué debe hacer, para qué lo hace y para quién; esto sólo es posible por medio de un exitoso proceso de comunicación interna con el que se da a conocer los pormenores de la institución, tomando en cuenta siempre que los trabajadores mejor 
informados forjan un alto compromiso con la organización (Cardozo Vale y Vásquez, 2014, p. 70), y que este compromiso, a su vez, cultiva la necesidad de pertenencia (Dávila de León y Jiménez García, 2014, p. 295). Esto refiere que la comunicación interna debe impulsar la motivación, la lealtad empresarial y el sentimiento de orgullo (Torres Huamanyauri, 2018, p. 12).

Por otro lado, $\mathrm{Ng}$ et al. (2019) expresan que el orgullo organizacional es el placer que siente el personal al estar asociado con la institución; abarca la medida en que los individuos experimentan una sensación de realización, derivada de su pertenencia organizacional (p. 110). Si un colaborador trabaja para una empresa con una buena reputación y estatus prestigioso en la sociedad, aumentará su orgullo de pertenencia a esta, lo que a su vez mejorará su comportamiento (Oo et al., 2018, p. 2).

El aumento en el compromiso del personal se ha considerado como una tarea destacada de la gestión de talento humano, pues los miembros del personal altamente comprometidos tienden a participar mayormente en los objetivos organizacionales, a compartir su visión, misión, valores y políticas (Yu et al., 2019, p. 965). Los colaboradores comprometidos no solo persiguen los objetivos relacionados con su trabajo, sino que también contribuyen plenamente al logro de los objetivos organizacionales, trabajando en equipo y asumiendo responsabilidades (Masri \& Suliman, 2019, p. 130); además, sienten gran motivación por aprender nuevas estrategias de trabajo, por desarrollar habilidades, por adaptarse al cambio y desarrollar comportamientos acordes a la cultura de trabajo (Endris Seid \& Dawit Negassa, 2019, p. 88).

Al respecto, la información que trasmite una organización repercute en la forma en la que los grupos de interés visualizan y se comprometen con la misma; en este sentido, un proceso de comunicación congruente reduce la aparición de información errónea (Cárdenas-Valencia \& Aguilar-Vélez, 2018).

En las organizaciones de servicios, un aspecto fundamental es la intangibilidad del producto, lo cual conlleva a que el marco de la comunicación se convierta en un componente vital en el proceso de construcción de una identidad institucional consolidada; por tanto, la comunicación interna es un instrumento esencial de las universidades para difundir su marca. Es así como una correcta gestión permitirá transmitir la identidad, de modo que refleje atracción; siendo menester que el mensaje emitido, a través de sus medios comunicacionales, sea claro para todo su público, tanto interno (estudiantes, docentes, personal administrativo) como externo; y este 
mensaje, a su vez, debe transmitir una imagen afín con su esencia (Takaki et al., 2015, p. 28). En este sentido, si el personal comprende íntegramente la identidad de la organización a la que pertenece, indudablemente esto se reflejará en la imagen que se figura de ella (Duque Oliva y Carvajal Prieto, 2015, p. 120).

Por otra línea, las herramientas de comunicación interna (correos, reuniones, entre otras) constituyen un factor clave para alcanzar los objetivos estratégicos, ya que su propósito es cuidar al cliente interno, servir de soporte en la creación de una sólida cultura organizacional y minimizar la aparición de rumores, propiciando así un clima de confianza y motivación (Cardozo Vale y Vásquez, 2014, pp. 70-71).

\section{Identidad institucional}

La identidad institucional es una forma de representar a una organización, sobre su esencia y razón de ser, a través de varias formas de comunicación organizacional (Abdullah y Semana Rabiah, 2018).

En la misma perspectiva, Villafañe Gallego (2008) sostiene que la identidad institucional es "el conjunto de rasgos y atributos que definen la esencia de la organización, en donde algunos son visibles y otros no" (p. 26).

Existen dos elementos esenciales para comprender la identidad: cultura y filosofía organizacional. La primera es el "alma" y describe lo que la organización verdaderamente es; la segunda es la "mente", y representa lo que quiere llegar a ser (Apolo et al., 2017, p. 528).

A través del comportamiento de la organización también se trasmite identidad institucional, en donde su público interno y externo, es quien puede evaluar lo que realiza la entidad. En organizaciones de servicios, la trasmisión de identidad institucional precisa del colaborador que la conforma, ya que este, por medio de sus acciones manifiesta los valores de la organización hacia el cliente; es así como resulta ser esencial en la construcción de la identidad institucional. En este marco, las acciones que lleve a cabo cualquier institución de educación superior deben manifestar sus valores, facilitar al profesorado los medios necesarios para el desarrollo de nuevos conocimientos y habilidades, así como ofrecer un mejor servicio, con el afán de generar una identidad institucional consolidada y atractiva para sus públicos (Takaki et al., 2015, p. 28). Con 


\section{Incidencia de la}

el pasar del tiempo, estas instituciones han adoptado una orientación centrada en el estudiante, con esto han adquirido identidades institucionales solidificadas y favorables. En consecuencia, los académicos sostienen la importancia de consolidar la identidad en la educación superior (Balmer et al., 2020, p. 630).

En este sentido, según lo indicado en la figura 1, "La identidad de la Universidad Politécnica Salesiana se comprende a sí misma como católica, salesiana y fundamentada en la apuesta pedagógica preventiva, crítica y socio-constructivista" (Universidad Politécnica Salesiana -UPS-, 2018, p. 21).

\section{Figura 1}

Identidad de la Universidad Politécnica Salesiana

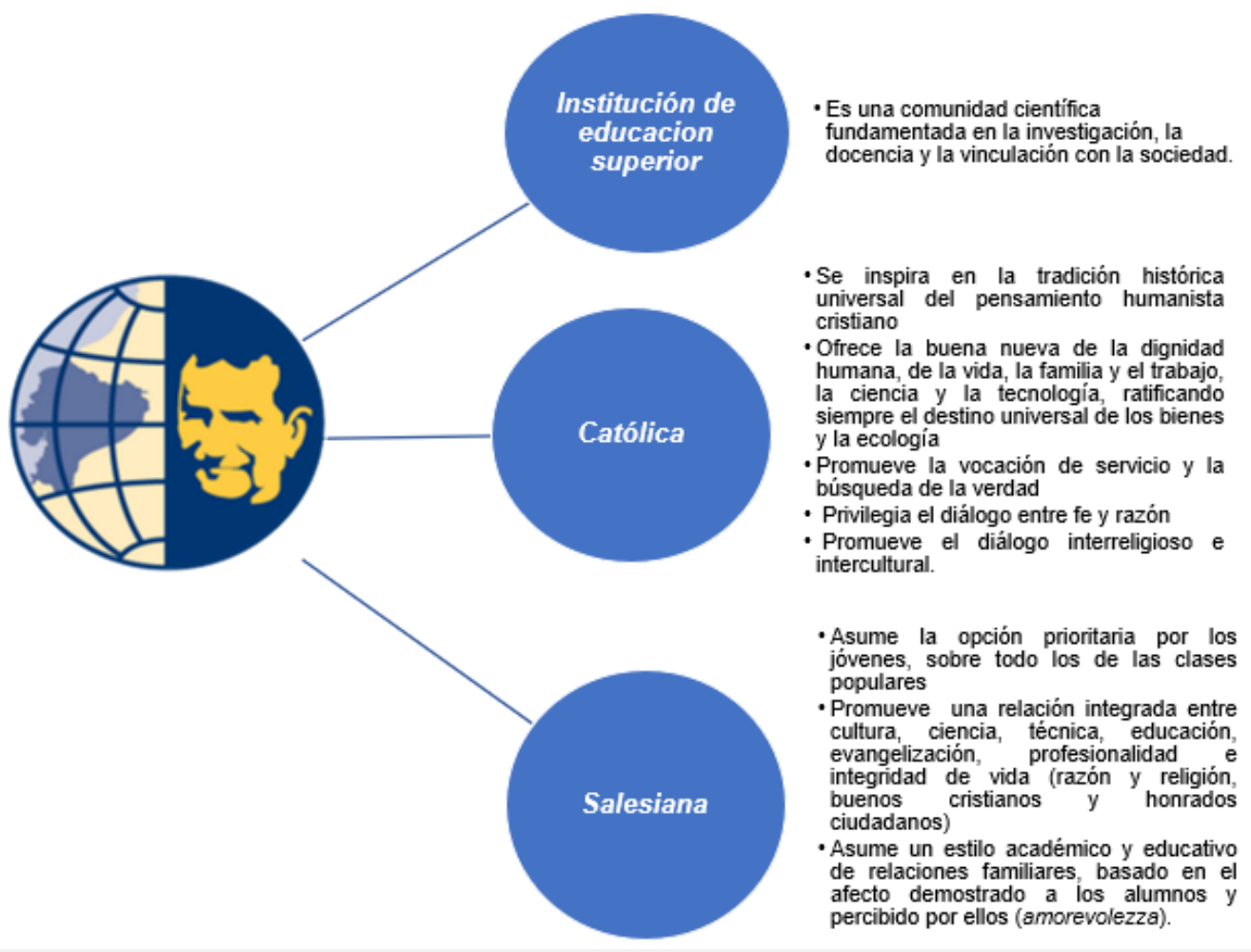

Nota: Elaboración propia, a partir de Universidad Politécnica Salesiana (2018).

Como parte de la identidad,

Uno de los componentes sustanciales del Modelo Pedagógico Salesiano de la UPS constituye el Sistema Preventivo que se lo conceptúa como un estilo para educar y orientar 
a los jóvenes a fin de que lleguen a ser "honrados ciudadanos y buenos cristianos" en base de conjugar la razón, religión y amabilidad, principios que indican una visión armónica de la persona. (...)

La razón, la religión y la amabilidad son tres dimensiones que fundamentan el Sistema Educativo Salesiano. Se plantean como ejes transversales porque atraviesan todo el proceso y las relaciones educativas. Los dos primeros términos (Razón - Religión) forman el contenido humano-cristiano de la educación. El tercer término (Amabilidad) se refiere a la metodología educativa (...) La transversalidad de la razón y la religión potencia en la Universidad Politécnica Salesiana el diálogo razón y fe desde la práctica de la amabilidad, las metodologías de enseñanza y aprendizaje.

Es un estilo de educar que pone como centro de la educación al educando y que prioriza relaciones de comunicación dialogadas y horizontales. La práctica de la amabilidad en el proceso educativo crea el clima de familiaridad con relaciones de bondad, sinceridad, respeto y cordialidad en el ambiente de aprendizaje. (UPS, 2018, pp. 23-24) En virtud del método preventivo de educación que maneja la Universidad como institución Salesiana propiciará y velará porque cada docente reciba la debida capacitación en dicho método, basado en el amor, la comprensión y sobre todo en principios éticos. Los docentes de la Universidad Politécnica Salesiana, son responsables de la "formación integral” de los graduados, como parte de la orientación fundamental dada para los perfiles profesionales, junto al contenido científico tecnológico y humanista dentro de una labor académica comprometida con el ejemplo y la vivencia de los valores, dónde prevalezca actitudes de respeto incondicional a la persona, equidad, justicia, franqueza, transparencia, honestidad, la búsqueda del bien común y el servicio a la sociedad de forma preferencial a los pobres. (UPS, 2015, p. 7)

\section{Cultura organizacional}

La cultura organizacional integra creencias, valores, actitudes o prácticas compartidas por el personal (Bąk \& Sukiennik, 2019, p. 1). 
Desde la misma perspectiva, la cultura organizacional es el cúmulo de creencias, valores y prácticas, que guían y reflejan el comportamiento del personal; a su vez, las creencias son el conjunto de supuestos compartidos, en relación con diversos temas; también, constituyen estructuras no notorias, instintivas y predeterminadas. Asimismo, los valores son principios compartidos por el personal en su convivir diario, son más tangibles que las creencias, empero, no se pueden observar con total claridad; $y$, finalmente, las prácticas son aquellas directrices notorias bajo las cuales trabaja el personal (Apolo et al., 2017, p. 529).

Simon \& Varghese (2018) sostienen que la cultura organizacional es un patrón de supuestos básicos compartidos, cuyos miembros de la empresa desarrollan y descubren por medio del aprendizaje (p. 350).

Por su parte, Abualoush et al. (2018) y Hernández-Castorena et al. (2018) afirman que la cultura organizacional es un factor vital para reforzar la gestión del conocimiento del personal, en su forma de aprender y compartir saberes (p. 282).

En general, la cultura organizacional refleja las reglas no escritas de una empresa; también, puede considerarse como una forma de propiedad intelectual o un activo intangible (Ketprapakorn \& Kantabutra, 2019, p. 3).

Tzianakopoulou \& Manesis (2018) señalan que la cultura organizacional constituye una característica fundamental de las instituciones educativas, debido a que influye en la forma de pensar y de comportarse por parte de sus miembros, así como en la productividad del personal y en el rendimiento académico del estudiante (p. 2519). Referente a esto, la cultura organizacional en:

El Código de Convivencia de la Universidad Politécnica Salesiana se fundamenta y asume el sistema de principios y valores que están inmersos en las características de ser una Universidad Católica y Salesiana. Es necesario identificar aquellos principios que emergen desde algunas miradas; en este caso, el rol como Universidad Salesiana y como profesor universitario de una Universidad Católica:

Un estilo académico y educativo de relaciones basado en el afecto demostrado a los estudiantes y percibido por ellos (amorevolezza).

Docentes que garanticen una manera y forma intelectual rigurosa y crítica, cuyas características son el resultado de la integración de los valores humanos y profesionales. 
Incidencia de la

comunicación
Incidencia de la comunicación | Revista Virtual

Universidad Católica del Norte, 63, 94-125

ISSN: 0124-5821 (En línea)

Ser testigo de una vida que integra la Fe y la Ciencia, la Fe y la Cultura, competencia profesional y sabiduría cristiana. (UPS, 2012, pp. 4-7).

\section{Filosofía organizacional}

La filosofía organizacional es la concepción general de la institución, determinada por la dirección como central, imperecedera y característica, que contribuye al logro de los objetivos (Apolo et al., 2017, p. 529). La filosofía organizacional se compone por: i) Misión, es la declaración que define la actividad de la organización, su razón de ser y operar. Para Hategan et al. (2019) la misión puede expresarse como: the firm 's belief o el credo de la institución, su propósito, entre otros (p. 2); ii) Visión, hace referencia a su perspectiva de futuro, es decir, lo que pretende lograr la organización a largo plazo; iii) Políticas, son directrices generales para orientar la acción de la organización; y iv) Valores centrales, son la acumulación de principios que guían a los colaboradores de una empresa; estos deben ir acorde con la identidad institucional (Leguado et al., 2018, pp. 3-4).

A pesar de que existen investigaciones que confirman que la visión, misión, políticas y valores organizacionales influyen en el desempeño del personal y de las empresas, es evidente que únicamente estos enunciados no son suficientes, sino que además se debe considerar que los colaboradores sientan el significado de esta filosofía, para que así puedan ejercerlos con pasión y positivismo; por tanto, no solo basta con definir dichos enunciados, sino que también resulta esencial difundirlos entre su público interno para que estos lleguen a adoptarlos y a reflejar en los públicos externos la esencia de la organización (Dermol \& Trunk Širca, 2018, p. 542).

Las declaraciones de la misión, visión, valores institucionales y políticas de comunicación de la Universidad Politécnica Salesiana son:

\section{Misión}

La Universidad Politécnica Salesiana es una institución de educación superior humanística y politécnica, de inspiración cristiana con carácter católico e índole salesiana; dirigida de manera preferencial a jóvenes de los sectores populares; busca formar "honrados 
Incidencia de la comunicación
Incidencia de la comunicación | Revista Virtual

Universidad Católica del Norte, 63, 94-125

ISSN: 0124-5821 (En línea)

ciudadanos y buenos cristianos", con excelencia humana y académica, con capacidad investigativa e innovadora, que contribuyan al desarrollo sostenible local y nacional.

\section{Visión}

Al 2023, la Universidad Politécnica Salesiana es una institución de educación superior de referencia en la búsqueda de la verdad y el desarrollo de la cultura, de la investigación científica y tecnológica; reconocida socialmente por su calidad en la academia y producción científica, por su responsabilidad social universitaria y por su capacidad de incidencia en la innovación, interculturalidad y el desarrollo.

\section{Valores institucionales}

La comunidad educativa de la Universidad Politécnica Salesiana fiel a la misión y visión prioriza los siguientes valores: Trascendencia, familiaridad, corresponsabilidad, solidaridad y honestidad. (UPS, 2018, pp. 27-28).

\section{Políticas de comunicación}

Las Políticas de Comunicación apoyan y promueven todas las acciones comunicativas internas y externas de la Universidad Politécnica Salesiana.

La organización y procesos de comunicación estarán en concordancia con la Misión y Visión institucionales, el proyecto orgánico y a las funciones propias de la Universidad Politécnica Salesiana.

Los procesos de comunicación se desarrollarán dentro del cuadro de referencia de la Pastoral Juvenil Salesiana, como dimensión de la acción educativo-pastoral, como obra y campo de misión, como gestión de procesos y productos destinados a crear y reforzar ambientes comunicativos salesianos. (UPS, 2011, pp. 1-2)

\section{Metodología}

En la presente investigación se aplicó la metodología hipotética-deductiva con alcance descriptivo y correlacional, debido al diagnóstico de la influencia de la comunicación interna en 
Incidencia de la comunicación
Incidencia de la comunicación | Revista Virtual

Universidad Católica del Norte, 63, 94-125

ISSN: 0124-5821 (En línea)

la identidad institucional del docente. El diseño fue de tipo mixto cuali-cuantitativo, puesto que se utilizaron las técnicas de investigación: análisis del discurso y el modelo de Teün Van Dijk, a través de la construcción del corpus textual, basado en la identificación de mensajes explícitos e implícitos de las comunicaciones emitidas por la Universidad Politécnica Salesiana, comprendidas entre los años 2017 y 2019. En derivación de esto, se procedió con el establecimiento de macroestructuras semánticas, que resumieran el sentido general del discurso, por medio de ideas principales. Adicionalmente, en abril y mayo de 2019 se llevaron a cabo entrevistas semiestructuradas, con muestreo no probabilístico, a siete personas entre autoridades y docentes, procesadas bajo la misma técnica. Para triangular la información se realizaron encuestas en mayo de 2019, previamente sometidas a revisiones de validez y consistencia, juicio de expertos y prueba piloto, obteniendo un alfa de Cronbach de 0.887 , lo que denotó que el instrumento fue fiable. La muestra probabilística estratificada estuvo conformada por 515 docentes de las tres sedes (Quito, Guayaquil y Cuenca) de la Universidad Politécnica Salesiana, proveniente de una población de 968 docentes (Tabla 1), utilizando un nivel de confianza del $95 \%$ y un error del $5 \%$. Con base en esto, el análisis de datos se realizó a través del programa estadístico IBM SPSS® Statics, versión 25.0; y la interpretación de los resultados se dio mediante discusiones participativas entre los autores.

\section{Tabla 1}

Muestra estratificada 2019

\begin{tabular}{lcc}
\hline & \multicolumn{2}{c}{ Docentes } \\
\hline Sede & Población & Muestra \\
\hline Quito & 443 & 205 \\
\hline Cuenca & 270 & 157 \\
\hline Guayaquil & 255 & 153 \\
\hline TOTAL & $\mathbf{9 6 8}$ & $\mathbf{5 1 5}$ \\
\hline
\end{tabular}

Nota: elaboración propia.

\section{Resultados}

Acorde a la técnica análisis del discurso propuesta por Van Dijk (2001) luego de la interpretación de los significados locales, cuyo contenido semántico se integra por mensajes 
Incidencia de la

comunicación
Incidencia de la comunicación | Revista Virtual

Universidad Católica del Norte, 63, 94-125

ISSN: 0124-5821 (En línea)

explícitos e implícitos, provenientes de las comunicaciones emitidas por la Universidad Politécnica Salesiana, se procede a establecer macroestructuras que resumen las ideas centrales de los textos analizados; estas se muestran a continuación:

En la Universidad Politécnica Salesiana, prima una tendencia hacia lograr una calidad académica sostenida. Existe un interés por la innovación de la Universidad Politécnica Salesiana a través de la eficacia. Marcado interés en desarrollar la docencia, investigación y vinculación con la sociedad para la consolidación del Modelo Educativo Pastoral Salesiano. Orientación hacia la conciliación de las estructuras de identidad salesiana, calidad académica y sostenibilidad. Reflexión permanente sobre los orígenes filosóficos de la obra salesiana. Enfoque o centralidad en el bienestar académico y humano del estudiante. Alto compromiso en el trabajo por la misión de la Universidad Politécnica Salesiana. Énfasis en la diversidad de la formación académica y pedagógica de los docentes de la institución para la universalidad.

Seguidamente se exponen los resultados de las entrevistas realizadas a través del descubrimiento de conceptos, categorías y patrones, presentes en los datos recolectados; los vínculos de otorgamiento de sentido para la interpretación están estrictamente relacionados con la metodología planteada.

A decir de los directivos entrevistados, las estrategias de comunicación que maneja la Universidad Politécnica Salesiana, para informar sobre filosofía y demás asuntos, son: eventos (académicos/sociales), reuniones, correos electrónicos y revistas; también, como parte de una comunicación ascendente, fluyen mensajes individuales hacia la dirección. Respecto a las estrategias para comunicar y fomentar identidad, la Secretaría Técnica del Aseguramiento de la Identidad Institucional y el rectorado expresan que mantienen encuentros presenciales con los docentes, cursos virtuales, diálogos con docentes de tradición salesiana, y artículos escritos sobre identidad en revistas institucionales: NotiUPS, Cuadernos de Reflexión Universitaria, entre otras. Adicional a esto, los entrevistados manifiestan que, para lograr transferir el sentimiento de pertenencia del docente hacia la Universidad Politécnica Salesiana, se han llevado a cabo cursos de identidad, a cargo de la misma Secretaría, además se intenta incorporar a los docentes con responsabilidades diversas y participativas, lo que conlleva a involucrarlos activamente en la toma de decisiones. 
Desde otro enfoque, para compatibilizar la academia con el Modelo Educativo Pastoral Salesiano, los directivos señalan que a los docentes se los anima a compartir, a formar claustros, a suplirse; el docente que no se acoge a este carisma salesiano, termina retirándose de la institución por cuenta propia, y la Universidad Politécnica Salesiana busca crear una conciencia comunicativa de que evidentemente no se apoya la gestión individualista. En cuanto a las divergencias pedagógicas, el rectorado afirma no preocuparle porque responden de cierta manera a los criterios y a la identidad del docente, y esa diversidad indudablemente enriquece a la Universidad Politécnica Salesiana. De igual forma, la dirección de la universidad mantiene que "el conocimiento no es de ahora, sino más bien que es de oportunidades”, y para sostener aquello, se ha implementado la reforma al "Reglamento Interno de Régimen Académico de la Universidad Politécnica Salesiana”, en cuanto a la evaluación de aprendizajes, que implica ofrecerle al estudiante diversas oportunidades en su preparación, a fin de que alcance el conocimiento planificado.

En otro aspecto, según los entrevistados, los símbolos identitarios más representativos entre los docentes son: el logotipo de la Universidad Politécnica Salesiana; Don Bosco y su lema: "Honrados ciudadanos y buenos cristianos"; la imagen de María Auxiliadora; así como el símbolo de las Instituciones Salesianas de Educación Superior (IUS), pues produce resonancia en la vinculación de la Universidad Politécnica Salesiana con otras instituciones universitarias salesianas en el ámbito internacional.

Adicionalmente, a decir de los entrevistados, las creencias, valores y prácticas que deben manejar los docentes como elementos básicos de la identidad institucional, giran en torno al Modelo Educativo Pastoral Salesiano; para tal efecto, las creencias deben estar fundamentadas en el estilo salesiano de preventividad, en la dignidad del estudiante y en el respeto a la identidad religiosa de la Universidad Politécnica Salesiana, que no necesariamente exige que todos quienes forman la comunidad universitaria sean católicos, porque se propende hacia la pluralidad y universalidad; empero, sí es necesario que se respete y se reconozca dicha identidad.

Por analogía, algunos de ellos creen que es posible ejercer la fe en el contexto de la educación con los jóvenes, y naturalmente esto implica conocer a Don Bosco, su obra, su pensamiento y su figura; aunque no todos están de acuerdo con esta vinculación, en vista de que consideran que la academia no deja espacio para este ejercicio; asimismo, creen que es importante 
tener la firme convicción de que el desarrollo de una sociedad tiene como base la formación de excelentes profesionales, con capacidad crítica y actitud proactiva, y que en dicha aseveración está intrínseco el deber de formar "Buenos cristianos y honrados ciudadanos".

En cuanto a los valores, según los entrevistados, estos se van creando, formando y se van apoyando con base en los valores universales, como dignidad, respeto, honradez, solidaridad, responsabilidad, libertad, creatividad, aceptación de riesgo y filiación institucional; con esto, la Universidad Politécnica Salesiana anima a que los docentes ejerzan valores acordes con el proyecto pastoral educativo, que más allá de formar académicamente al estudiante, se desarrolle al ser humano de manera integral.

En lo concerniente a las prácticas que deben manejar los docentes, los entrevistados expresan que están vinculadas directamente a los valores; puesto que, lo que se pretende como universidad es que los docentes se integren y que trabajen en la construcción de una academia en búsqueda constante de respuestas a las exigencias de los nuevos contextos universitarios, de acuerdo con el estilo preventivo, con prácticas académicas, investigativas, pero también humanas, cristianas y salesianas.

La Universidad Politécnica Salesiana como institución no establece prácticas, al contrario, estas se van creando como una espiral de conocimiento que da vueltas y que no permanece rígida. A la par, los docentes entrevistados expresan que es importante en el rol de educador, ejercer dentro de las prácticas, la evaluación de contenidos con base en el pensamiento de Don Bosco; es decir, asumirla como parte del proceso formativo humano y profesional, mas no como un medio de castigo, venganza o desde una tendencia a la minimización del número de evaluaciones al estudiante; y con base en este tema, surge la reforma al "Reglamento Interno de Régimen Académico de la Universidad Politécnica Salesiana" que, a consideración de los entrevistados, consiste en entender la evaluación desde la raíz de la filosofía organizacional; es decir, no condenar a nadie a priori por un error o por distintas situaciones sociales, económicas, académicas, emocionales, entre otras, sino más bien otorgarle otra oportunidad de demostrar sus conocimientos. No obstante, algunos entrevistados también afirman que existió un déficit de comunicación con algunos estudiantes y docentes, puesto que, en ciertos ambientes de la universidad, circulan comentarios negativos, como que esta medida ha dado cabida a facilismos, y así surgen grandes dudas en los entrevistados sobre ¿Cómo los estudiantes llegaron a conocer esta disposición?, ¿de 


\section{Incidencia de la} comunicación
Incidencia de la comunicación | Revista Virtual

Universidad Católica del Norte, 63, 94-125

ISSN: 0124-5821 (En línea)

qué forma fue presentada?, debido a que algunos consideran que el docente tiene la obligación de "aprobarlos en la materia que cursan"; lo que a criterio de varios entrevistados desvirtúa totalmente el sentido de la práctica propuesta por la universidad.

Respecto a las encuestas realizadas, se plantea un cuestionario conformado por 14 preguntas, que surgen con base en los datos obtenidos de las entrevistas y del análisis del discurso. Los resultados más relevantes son:

La figura 2 señala que el canal de comunicación con mayor impacto para el conocimiento de la filosofía de la Universidad Politécnica Salesiana es el correo electrónico; en referencia a los medios con mayor efectividad que fomentan la identidad institucional, están los encuentros presenciales, seguido de los diálogos con docentes de tradición salesiana.

\section{Figura 2}

Impacto y efectividad de canales de comunicación de la Universidad Politécnica Salesiana.

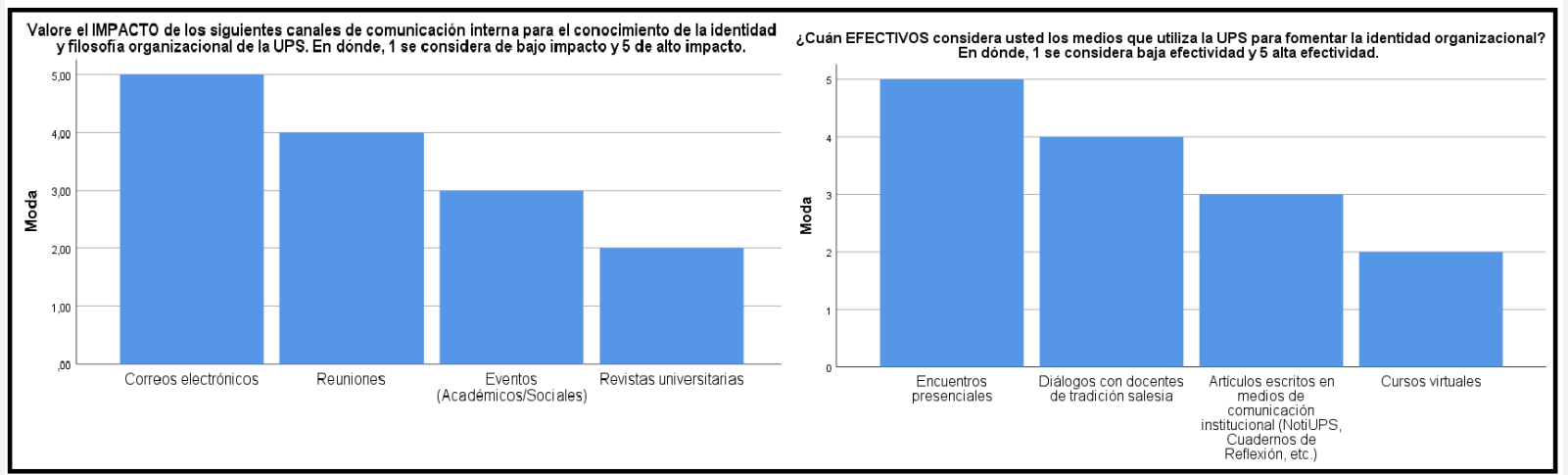

Nota: Elaboración propia.

Desde otra variable, alrededor del $50 \%$ de los docentes afirma que la comunicación institucional motiva frecuentemente para el desarrollo de la investigación científica y tecnológica dentro de las aulas, el desarrollo de proyectos de vinculación con la sociedad, y la formación de claustros docentes, grupos de investigación e innovación educativa (Véase figura 3). 


\section{Incidencia de la} comunicación
Incidencia de la comunicación | Revista Virtual

Universidad Católica del Norte, 63, 94-125

ISSN: 0124-5821 (En línea)

\section{Figura 3}

Motivación comunicacional de la Universidad Politécnica Salesiana hacia los docentes

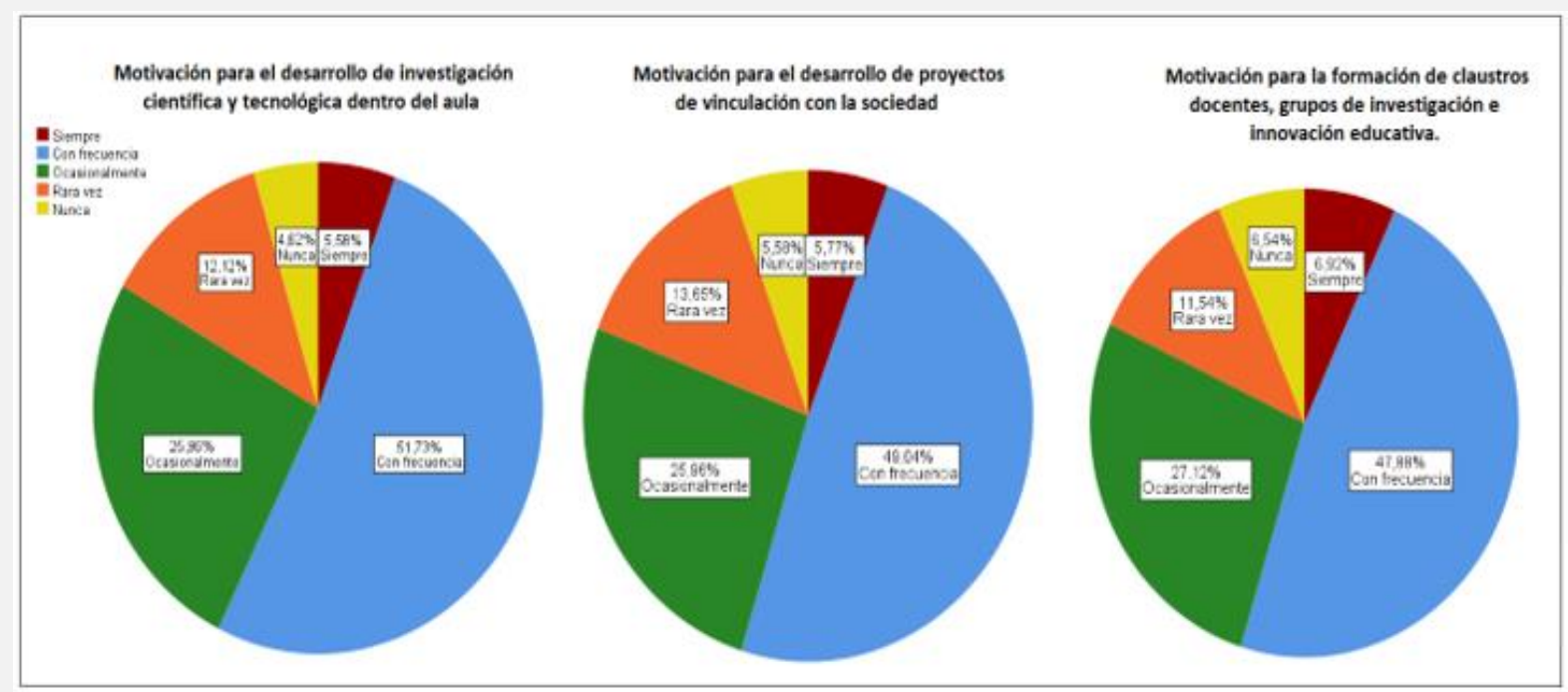

Nota: Elaboración propia.

En la figura 4, se evidencia que el $60 \%$ de los docentes (suma de 33\%, $20 \%$ y $7 \%$ ) ha comprendido que la reforma al "Reglamento interno de régimen académico de la Universidad Politécnica Salesiana", en cuanto a la evaluación de aprendizajes, responde a la filosofía organizacional.

\section{Figura 4}

Comprensión de la reforma al "Reglamento Interno de Régimen Académico de la Universidad Politécnica Salesiana" 


\section{Incidencia de la} comunicación
Incidencia de la comunicación | Revista Virtual

Universidad Católica del Norte, 63, 94-125

ISSN: 0124-5821 (En línea)

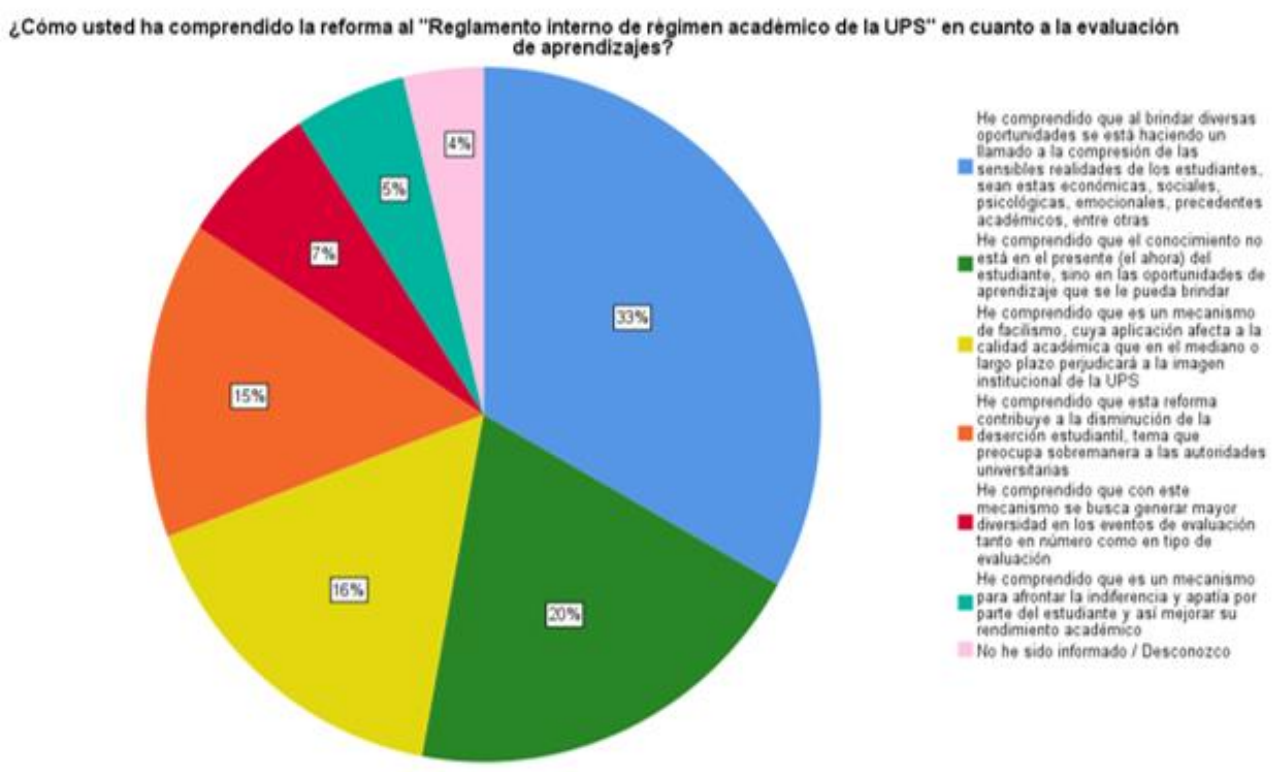

Nota: Elaboración propia.

Desde otro enfoque, la figura 5 indica que el $13 \%$ de los docentes considera que la Universidad Politécnica Salesiana siempre ha comunicado lo que pretende el Modelo Educativo Pastoral Salesiano, mientras que un $54 \%$ considera que se lo ha realizado frecuentemente.

\section{Figura 5}

Comunicación del Modelo Educativo Salesiano.

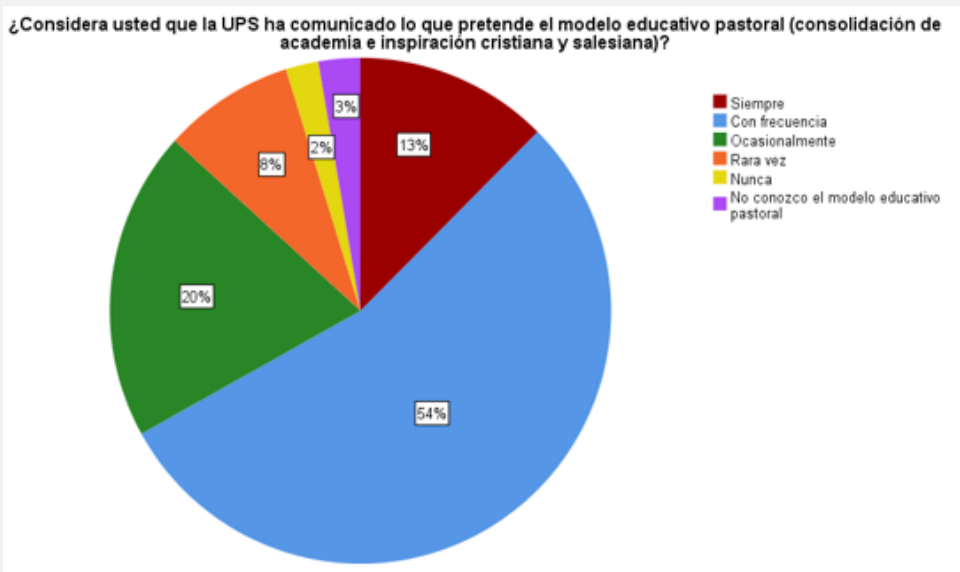

Nota: Elaboración propia. 


\section{Incidencia de la} comunicación
Incidencia de la comunicación | Revista Virtual

Universidad Católica del Norte, 63, 94-125

ISSN: 0124-5821 (En línea)

A su vez, como se muestra en la figura 6, en general todos los docentes consideran que el Modelo Educativo Pastoral de la Universidad Politécnica Salesiana promueve una educación de calidad, debido a que ofrece una formación integral basada en el constructivismo, en la investigación e innovación y en la inclusión educativa.

\section{Figura 6}

Modelo Educativo Salesiano.

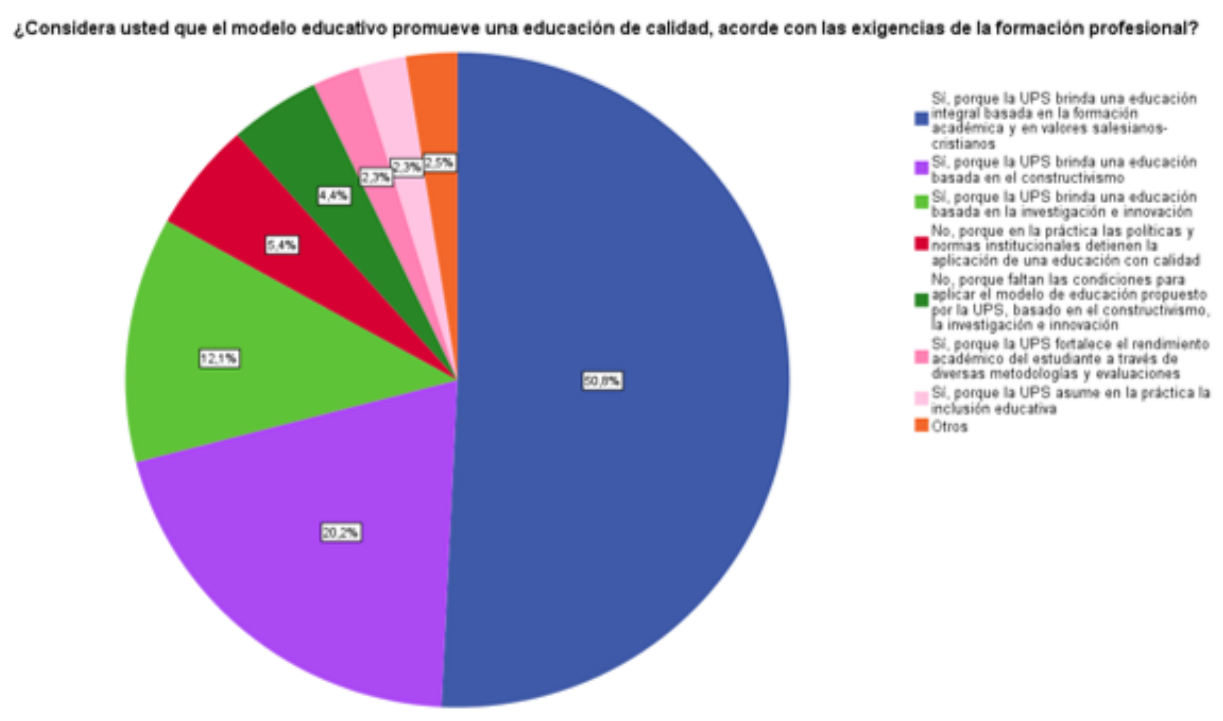

Nota: Elaboración propia.

En otro orden, al observar la figura 7, que resume la frecuencia de aplicación en la práctica docente sobre máximas institucionales tomadas textualmente, tales como: "La educación es cuestión del corazón", "Educamos evangelizando y evangelizamos educando", "Formamos honrados ciudadanos y buenos cristianos", "Nos fundamentamos en un Modelo Educativo orientado a la vinculación con la sociedad" y "Promovemos la investigación con identidad, calidad, sostenibilidad e innovación", se afirma que el $90 \%$ de los docentes (suma de $62 \%$ y $28 \%$ ) asume la identidad institucional. 


\section{Incidencia de la}

\section{comunicación}

Incidencia de la comunicación | Revista Virtual

Universidad Católica del Norte, 63, 94-125

ISSN: 0124-5821 (En línea)

\section{Figura 7}

Identidad institucional del docente de la UPS.

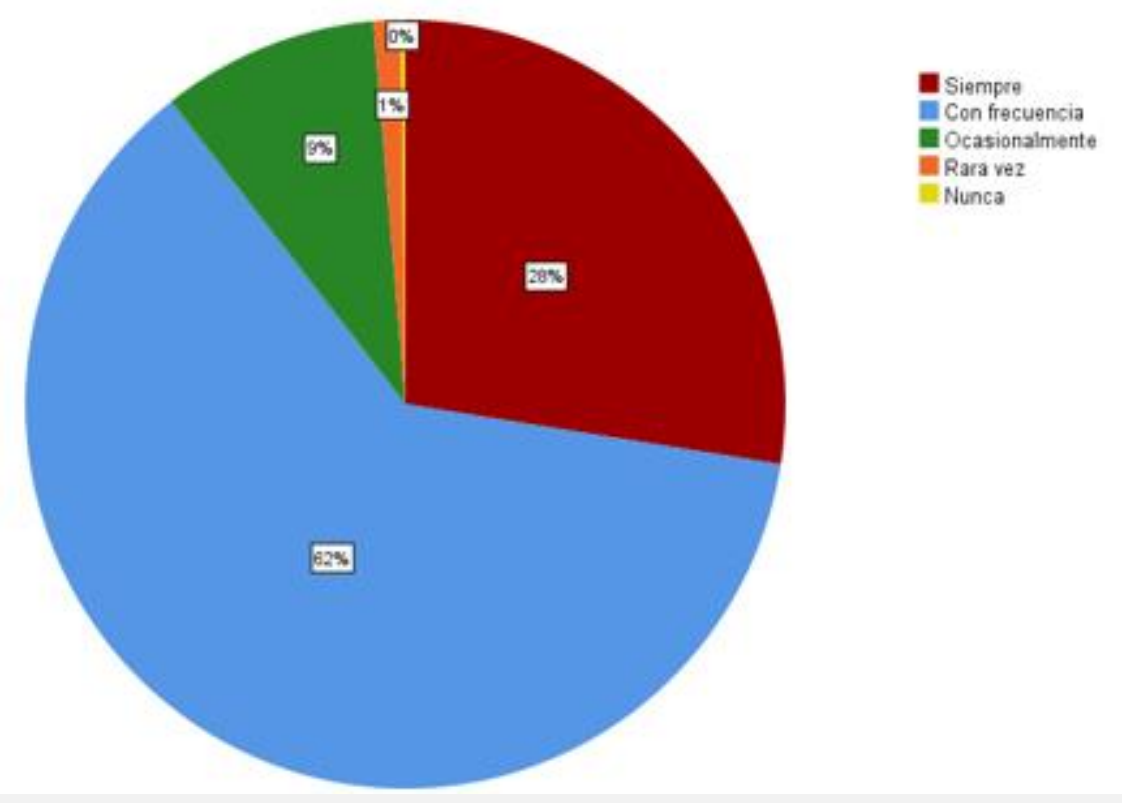

Nota: Elaboración propia.

Desde otro ángulo, la figura 8 ilustra que el $91 \%$ de los docentes (suma de $74 \%$ y $17 \%$ ) siente un elevado orgullo de pertenencia por la Universidad Politécnica Salesiana; este resultado, al ser comparado con las sedes estudiadas, muestra que $86 \%$ y $87 \%$ de los docentes pertenecientes a las sedes Cuenca y Guayaquil, respectivamente, son quienes sienten mayor orgullo, a diferencia de un $54 \%$ de docentes de la sede Quito. 


\section{Incidencia de la}

comunicación
Incidencia de la comunicación | Revista Virtual

Universidad Católica del Norte, 63, 94-125

ISSN: 0124-5821 (En línea)

\section{Figura 8}

Orgullo de pertenencia a la UPS

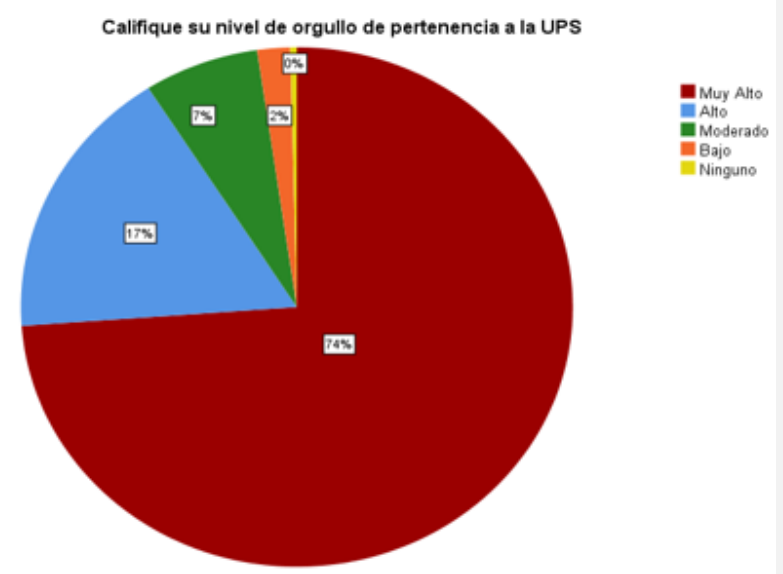

Orgullo de pertenencia a la Universidad Politécnica Salesiana por sedes

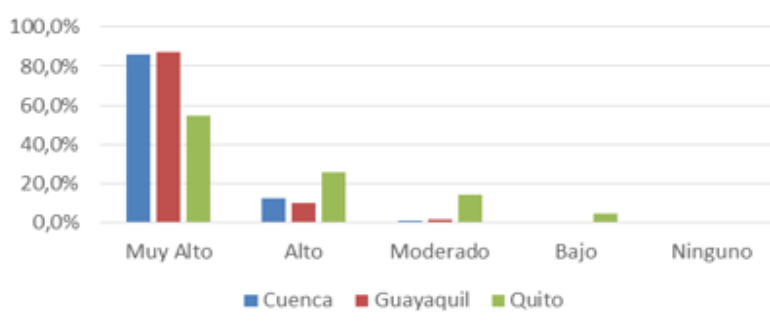

Nota: Elaboración propia.

Desde otra perspectiva, partiendo de la pregunta realizada a los docentes, acerca del origen diverso de su formación humana, el resultado muestra que el $83 \%$ se adapta sin limitantes al Modelo Educativo Pastoral Salesiano; es decir, sus creencias, valores y prácticas son congruentes con la filosofía y cultura institucional.

En lo relacionado con la hipótesis planteada: "La comunicación interna incide en la identidad institucional del docente de la Universidad Politécnica Salesiana", y su hipótesis nula: "La comunicación interna no incide en la identidad institucional del docente de la Universidad Politécnica Salesiana", al ser sometidas a la prueba no paramétrica de Kruskal-Wallis, que contrasta "la comunicación del Modelo Educativo Pastoral Salesiano" con "la frecuencia de aplicación en la práctica docente sobre máximas institucionales", se evidencia que el valor p es de 0.00, inferior al nivel de significación 0.05 , lo que conlleva a rechazar la hipótesis nula; por tanto, 
Incidencia de la

comunicación
Incidencia de la comunicación | Revista Virtual

Universidad Católica del Norte, 63, 94-125

ISSN: 0124-5821 (En línea)

se acepta que la comunicación interna incide en la identidad institucional del docente de la Universidad Politécnica Salesiana.

\section{Discusión}

El presente estudio tiene implicaciones de interés prácticas, asociadas a la gestión de identidad institucional en el ámbito de la educación superior y a la investigación académica, orientado desde un pensamiento vitalista, que aporta bases cuantitativas y cualitativas para la toma de decisiones en la institución, sujeto de estudio. Es así que desde el enfoque de la identidad y comunicación organizacional, se involucra el análisis de diálogos, de cuyo contenido semántico se establecen macroestructuras marcadas por la calidad académica, el bienestar humano del estudiante, la diversidad en formación académica y pedagógica de los docentes, el interés por la innovación a través de la eficacia y el desarrollo de la docencia, investigación y vinculación con la sociedad; lo que conllevaría a la consolidación del Modelo Educativo Pastoral Salesiano y a su sostenibilidad en el tiempo; por tanto, los resultados son singulares y al ser contrastados con estudios similares, se pretende sean la base para investigaciones más generalizadas.

El análisis de las variables de estudio connota que los encuentros presenciales y los diálogos con docentes de tradición salesiana son los medios más efectivos para fomentar la identidad universitaria asociada con la misión institucional (véase figura 2); desde ahí, se puede deducir que la identidad de la Universidad Politécnica Salesiana se sostiene con la participación activa del docente, debido a que, los artículos escritos en medios de comunicación institucional no presentan gran impacto en este aspecto; esta afirmación va acorde con los hallazgos del estudio de Takaki et al. (2015), quienes sostienen que los elementos escritos, que implican la comunicación de la identidad institucional de la universidad, no ejercen una influencia considerable en la identidad percibida por los docentes en las diferentes universidades estudiadas.

Desde otro matiz de la investigación, se demuestra que a la fecha del estudio, un $50 \%$ de los docentes opina ser motivado frecuentemente por la comunicación para: la investigación científica y tecnológica dentro de las aulas, el desarrollo de proyectos de vinculación con la sociedad, y la formación de claustros docentes, grupos de investigación e innovación educativa; y, al examinar con mayor detalle los resultados, se identifica que entre sedes existen diferencias; así, 
los docentes de las sedes Cuenca y Guayaquil son quienes están mayormente incididos por la comunicación, existiendo una significativa disparidad con la sede Quito; por lo que se sostiene que es necesaria una dinamización de los procesos comunicacionales para la optimización de las funciones sustantivas de la educación superior en la universidad, fundamentalmente en la sede Quito. Estos resultados están alineados con lo obtenido en otros trabajos, como el de Cuenca Lalangui (2017), en el que se destaca la opinión del Coordinador académico de la Universidad Politécnica Salesiana, sede Quito, específicamente de la carrera Administración de Empresas, quien considera que no se realiza una comunicación completa, únicamente se emite información; por lo que es necesario intensificar los procesos comunicativos y no enfocarse solamente en socializar eventos o noticias. Cabe recalcar que frente al estudio de Cuenca Lalangui (2017), esta investigación ofrece una perspectiva identitaria y comunicacional que integra a todas las sedes y carreras de Universidad Politécnica Salesiana, y no únicamente a una fracción de ella.

Siguiendo el análisis, los datos de la variable de control diseñada (véase figura 8), muestran que el $91 \%$ de los docentes siente un elevado orgullo de pertenencia hacia la Universidad Politécnica Salesiana; es así que, partiendo de dos posiciones: primero, tomando como acotación la posición de $\mathrm{Ng}$ et al. (2019), quienes refieren que el orgullo del personal surge cuando la organización se comunica, a fin de que sus colaboradores reciban información idónea que les permite evaluar su membresía organizacional desde una perspectiva positiva (p. 110); y, segundo, siguiendo los hallazgos de Bravo Gil et al. (2016), quienes infieren que una adecuada gestión de la identidad institucional, determina pautas de conducta como el orgullo de pertenencia hacia una organización (p. 59). Se confirma, por tanto, que la labor de la Universidad Politécnica Salesiana en este eje ha ejercido un rol fundamental para los resultados alcanzados. Esta variable de control es consistente con los datos obtenidos en el ítem que indaga la motivación comunicacional, descrita en el párrafo anterior, ya que se observa, también, que existe disparidad entre sedes.

Otro aspecto de importancia, lo constituye la necesidad de formalizar la comunicación ascendente en la institución; siendo así, el gran desafío de la Universidad Politécnica Salesiana mantener un equilibrio dinámico en la comunicación que se realiza, para fortalecer los vínculos entre colaboradores y la dirección de la institución, que logre potenciar un balance positivo en la construcción de una identidad institucional consolidada, así como para inspirar a que los docentes, 
en su totalidad, se sientan expresivamente orgullosos de formar parte de una institución que trabaja para agregar valor a la sociedad.

Por otra parte, al analizar los resultados de las entrevistas y de las encuestas respecto, a la comprensión de la reforma al "Reglamento Interno de Régimen Académico de la Universidad Politécnica Salesiana" concerniente a la evaluación de aprendizajes, estos reflejan que el $60 \%$ de los docentes ha comprendido que estas prácticas están en congruencia con la filosofía organizacional; se puede afirmar, entonces, que no es un problema de comunicación entre directivos y docentes, pues un mayoritario porcentaje ha vislumbrado el verdadero sentido de esta praxis evaluativa; al contrario, los resultados resaltan que las percepciones diferentes a esta interpretación, se dan por un desfase de comunicación con los estudiantes, debido a su forma de comprensión en cuanto a la medida propuesta; por tanto, sería importante reorientar la comunicación de esta reforma con campañas de socialización, tanto para los estudiantes mal informados, como para el $16 \%$ del personal docente que no ha recibido una comunicación correcta de los fines del reglamento; dado que según los hallazgos de Ma (2018), el éxito de los esfuerzos estratégicos de comunicación con los públicos externos requiere necesariamente el apoyo de todos los públicos internos, pues son ellos quienes comunican al exterior lo que es en realidad la organización (p. 52); es así como una forjada comunicación fortalece la imagen institucional (Awan et al., 2018, p. 421).

Otra variable de estudio evidencia que el $83 \%$ de los docentes se sienten significativamente identificados con la visión, misión, valores y políticas de la institución, por lo que más allá de definir dichos enunciados, la Universidad Politécnica Salesiana se ha encargado de difundirlos entre su público interno (docentes), de forma que estos han llegado a adoptarlos; este resultado va de la mano con los hallazgos de la investigación de Dermol \& Trunk Širca (2018), quienes establecen, en su estudio, que no es suficiente con determinar la filosofía organizacional; al contrario, resulta esencial divulgarla al público interno, para que estos se apropien y reflejen en el público externo la esencia de la organización. 


\section{Conclusiones}

El objetivo del presente estudio es identificar la incidencia de la comunicación interna en la identidad institucional del docente de la Universidad Politécnica Salesiana; al respecto, los resultados llevan a inferir que la filosofía y cultura organizacional efectivamente sí respaldan la actual identidad institucional; esto se observa en el $90 \%$ de docentes que afirman aplicar las máximas institucionales, cuya praxis define la identidad de la universidad; mostrando así, que una adecuada gestión de los elementos que la componen, unidos a una dinámica comunicación, forjan mayor apropiación y vinculación del docente con su universidad.

En la misma línea de pensamiento, en cuanto a la cultura y filosofía organizacional asumidas por los docentes, el $83 \%$ afirma que sus creencias, valores y prácticas son congruentes con el Modelo Educativo Salesiano de la universidad; debido a que sostienen las funciones de docencia, innovación, investigación y vinculación con la sociedad, al estilo salesiano de preventividad.

Por otro lado, en la variable comunicación institucional, se infiere que alrededor del $50 \%$ de los docentes afirma que esta comunicación motiva frecuentemente para el desarrollo de la investigación, innovación educativa y proyectos de vinculación con la sociedad.

Finalmente, los resultados encontrados en el análisis del discurso y en las entrevistas, permiten apoyar la validez de la hipótesis planteada que contrasta "la comunicación del Modelo Educativo Pastoral Salesiano" con "la frecuencia de aplicación en la práctica docente sobre máximas institucionales", lo que conlleva a inferir que la comunicación interna efectivamente sí incide en la identidad institucional del docente de la Universidad Politécnica Salesiana; por tanto, es importante que la institución continúe consolidando su identidad, en relación con sus procesos comunicacionales, y lograr así seguir siendo reconocida, tanto desde la perspectiva del docente, que al momento alcanza alrededor de un $90 \%$, como para el público externo, por su propuesta identitaria de calidad, bajo un Modelo Educativo Pastoral Salesiano que se orienta a ofrecer una educación integral, basada en la formación académica y humana, en la innovación, la investigación y la vinculación con la sociedad. 
Incidencia de la

comunicación
Incidencia de la comunicación | Revista Virtual

Universidad Católica del Norte, 63, 94-125

ISSN: 0124-5821 (En línea)

\section{Referencias}

Abadía Sánchez, H., y Vaca López, A. (2012). La auditoría de comunicación: Una evaluación crítica de la efectividad de la comunicación organizacional en las Universidades Colombianas. Textos \& Sentidos, 6, 35-50. https://bit.ly/31LYIAd

Abdullah, Z., \& Seman Rabiah, R. A. (2018). Constructing a unique online corporate identity of Korean companies. 3rd International Conference on Applied Science and Technology ICAST, 2018(1), 020004-1-020004-5. http://doi.org/10.1063/1.5055406

Abualoush, S., Masa'deh, R., Bataineh, K., \& Alrowwad, A. (2018). The role of knowledge management process and intellectual capital as intermediary variables between knowledge management infrastructure and organization performance. Interdisciplinary Journal of Information, Knowledge, and Management, 13, 279-309. http://doi.org/10.28945/4088

Apolo, D., Baéz, V., Pauker, L., y Paquel, G. (mayo, 2017). Gestión de Comunicación Corporativa: consideraciones para el abordaje de su estudio y práctica. Revista Latina de Comunicación Social, 72, 521-539. http://doi.org/10.4185/RLCS-2017-1177

Awan, H. M., Hayat, S., \& Faiz, R. (2018). Antecedents and consequences of corporate image: Conventional and islamic banks. Revista de Administração de Empresas RAE, 58(4), 418432. http://doi.org/10.1590/S0034-759020180407

Bąk, P., \& Sukiennik, M. (2019). Impact of Corporate Culture on Business Goals of Energy Sector Companies. 2nd International Conference on the Sustainable Energy and Environmental Development SEED 2017, 1-6. http://doi.org/10.1088/1755-1315/214/1/012066

Balmer, J. M., Mahmoud, R., \& Chen, W. (2020). Impact of multilateral place dimensions on corporate brand attractiveness and identification in higher education: Business school insights. Journal of Business Research, 116, 628-641. http://doi.org/10.1016/j.jbusres.2019.03.0165

Brandão, N. G. (2018). A comunicação interna estratégica como reforço da valorização das pessoas e seus níveis de engagement nas organizações. Media and Jornalismo, 18(33), 91102. http://doi.org/10.14195/2183-5462_33_6 
Bravo Gil, R., Matute Vallejo, J., y Pina Pérez, J. (2016). Gestión de la identidad corporativa: Evidencias en el sector bancario. Revista de Ciencias Sociales, 22(2), 49-62. https://bit.ly/2Ymaxc1

Cárdenas-Valencia, L. E., y Aguilar-Vélez, B. D. (2018). Incidencia de la comunicación interna con los estudiantes del programa Ser Pilo Paga de la Universidad Nacional de Colombia, sede Medellín. Revista CEA, 4(8), 35-49. https://doi.org/10.22430/24223182.1046

Cardozo Vale, S., y Vásquez, M. (2014). Herramientas de comunicación interna en la Universidad de Los Andes, Trujillo. Visión Gerencial, 2014(1), 63-80. https://bit.ly/2Yzaxa5

Cea Esteruelas, M. N. (2018). Responsabilidad social universitaria: el papel de los medios de comunicación y sus stakeholders. Retos Revista de Ciencias de la Administración y Economía, 8(16), 95-105. http://doi.org/10.17163/ret.n16.2018.07

Cuenca Lalangui, M.V. (2017). Estudio de la percepción de la imagen institucional de la Universidad Politécnica Salesiana, sede Quito en los estudiantes de Administración de Empresas (Tesis de maestría). Universidad Andina Simón Bolívar.

Dávila de León, C., y Jiménez García, G. (junio, 2014). Sentido de pertenencia y compromiso organizacional: Predicción del bienestar. Revista de Psicología, 32(2), 273-302. https://doi.org/10.18800/psico.201402.004

Dermol, V., \& Trunk Širca, N. (2018). Communication, Company Mission, Organizational Values, and Company Performance. Procedia - Social and Behavioral Sciences, 238, 542551. http://doi.org/10.1016/j.sbspro.2018.04.034

Duque Oliva, E. J., y Carvajal Prieto, L. A. (2015). La identidad organizacional y su influencia en la imagen: una reflexión teórica. Suma de Negocios, 6(13), 114-123. http://doi.org/10.1016/j.sumneg.2015.08.011

Eltanskaya, E., Arzhanovskaya, A., \& Linkova, Y. (2019). Melodic features of emphatic apology speech formulae in business communication. 1st International Scientific Practical Conference on Breakthrough Technologies and Communications in Industry BTCI 2018, 483(1), 1-5. http://doi.org/10.1088/1757-899X/483/1/012040

Endris Seid, K., \& Dawit Negassa, G. (2019). Academic Staffs' Level of Organizational Commitment in Higher Educational Setting: The Case of Haramaya University. 
Incidencia de la

comunicación
Incidencia de la comunicación | Revista Virtual

Universidad Católica del Norte, 63, 94-125

ISSN: 0124-5821 (En línea)

International Journal of Higher Education, 8(2), 87-100. http://doi.org/10.5430/ijhe.v8n2p87

Garnica Gómez, L. (2011). Evaluación y medición de la gestión de la comunicación en las organizaciones empresariales colombianas. Revista Virtual Universidad Católica del Norte, (34), 406-430. https://bit.ly/2YD9Lc6

Hategan, C. D., Curea Pitorac, R. L., \& Hategan, V.P. (2019). The Romanian family businesses philosophy for performance and sustainability. Sustainability, 11(6), 1-22. http://doi.org/10.3390/su11061715

Hernández-Castorena, O., Colín-Salgado, M., y Velásquez-Espinosa, N. (2018). Análisis de la relación hábitos y aceptación de 9's como elemento de competitividad en la pyme manufacturera de Aguascalientes, México. Revista CEA, 4(7), 29-47. https://doi.org/10.22430/24223182.757

Ketprapakorn, N., \& Kantabutra, S. (2019). Culture Development for Sustainable SMEs: Toward a Behavioral Theory. Sustainability, 11(9), 1-15. http://doi.org/10.3390/su11092629

Leguado, R. I, Florez, E. G., \& Hernández, F. Y. (2018). Organizational philosophy as a vehicle of action in the competitive development of SMEs. 4th International Meeting on Applied Sciences and Engineering EISI 2018, 1126(1), 1-6. http://doi.org/10.1088/1742$\underline{6596 / 1126 / 1 / 012067}$

Ma, L. (2018). I am one of them: Examining crisis communication from an intergroup communication approach. Journal of Contingencies and Crisis Management, 27(1), 50-60. http://doi.org/10.1111/1468-5973.12232

Masri, N. E., \& Suliman, A. (2019). Talent Management, Employee Recognition and Performance in the Research Institutions. Studies in Business and Economics, 14(1), 127-140. http://doi.org/10.2478/sbe-2019-0010

Ng, T. W., Yam, K. C., \& Aguinis, H. (2019). Employee perceptions of corporate social responsibility: Effects on pride, embeddedness, and turnover. Personnel Psychology, 72(1), 107-137. http://doi.org/10.1111/peps.12294

Oo, E. Y., Jung, H., \& Park, I. J. (2018). Psychological Factors Linking Perceived CSR to OCB: The Role of Organizational Pride, Collectivism, and Person-Organization Fit. Sustainability, 10(7), 1-16. http://doi.org/10.3390/su10072481 
Incidencia de la

comunicación
Incidencia de la comunicación | Revista Virtual

Universidad Católica del Norte, 63, 94-125

ISSN: 0124-5821 (En línea)

Pérez, A., y Rodríguez del Bosque, I. (2014). Identidad, imagen y reputación de la empresa: integración de propuestas teóricas para una gestión exitosa. Cuadernos de Gestión, 14(1), 97-126. http://doi.org/10.5295/cdg.130389ap

Perozo de Jiménez, G. (2006). La gestión comunicacional basada en la cultura organizacional e identidad corporativa de la Universidad Nacional Abierta (UNA). Revista de Ciencias Sociales, 12(2), 363-364. https://bit.ly/3ox13qw

Pineda de Alcazar, M. (2001). Las teorías clásicas de la comunicación: Balance de sus aportes y limitaciones a la luz del siglo XXI. Opción. Revista de Ciencias Humanas y Sociales, 1129. https://bit.ly/2YzDL8S

Robbins, S. P., y Judge, T. A. (2017). Comportamiento organizacional. Pearson Education Inc.

Schoeneborn, D., Kuhn, T., \& Kärreman, D. (2019). The Communicative Constitution of Organization, Organizing, and Organizationality. Organization Studies, 40(4), 475-496. http://doi.org/10.1177/0170840618782284

Simon, S. M., \& Varghese, K. (2018). Assessment of organizational culture in construction - A case study approach. 26th Annual Conference of the International Group for Lean Construction: Evolving Lean Construction Towards Mature Production Management Across Cultures and Frontiers IGLC 2018, (1), 348-357. http://doi.org/10.24928/2018/0396

Stolyarchuk, L. I., Stolyarchuk, I. A., \& Chumakov, V. I. (2019). Gender-related specifics of communication in the workplace. 1st International Scientific Practical Conference on Breakthrough Technologies and Communications in Industry BTCI 2018(483), 1-7. http://doi.org/10.1088/1757-899X/483/1/012063

Takaki, M., Bravo, R., y Martínez, E. (abril, 2015). La gestión de la identidad corporativa en la Universidad: análisis y consecuencias desde la perspectiva del profesorado. Revista Europea de Dirección y Economía de la Empresa, 24(1), 25-34. http://doi.org/10.1016/j.redee.2014.05.001

Torres Huamanyauri, Y. E. (2018). Relación entre la eficacia de la comunicación interna y la identidad corporativa en los trabajadores del Área de Dirección de Promoción del Empleo y Capacitación Laboral del Ministerio de Trabajo (Tesis de pregrado). Universidad César Vallejo. 
Incidencia de la comunicación
Incidencia de la comunicación | Revista Virtual

Universidad Católica del Norte, 63, 94-125

ISSN: 0124-5821 (En línea)

Tzianakopoulou, T., \& Manesis, N. (2018). Principals' perceptions on the notion of organizational culture: The case of greece. Universal Journal of Educational Research, 6(11), 2519-2529. http://doi.org/10.13189/ujer.2018.061117

Universidad Politécnica Salesiana -UPS-. (2011). Políticas de comunicación de la Universidad Politécnica Salesiana. https://bit.ly/2YgbEKa

Universidad Politécnica Salesiana -UPS-. (2012). Código de Convivencia de la Universidad Politécnica Salesiana. https://bit.ly/2XBKjEU

Universidad Politécnica Salesiana -UPS-. (2015). Código de Ética de la Universidad Politécnica Salesiana. https://bit.ly/2J5iOdQ

Universidad Politécnica Salesiana -UPS-. (2018). Plan Estratégico Institucional - Carta De Navegación 2019 - 2023. https://bit.ly/2XxvZgQ

Van Dijk, T. (2001). Critical discourse analysis. En D. Schiffrin, D. Tannen \& H. E. Hamilton (Eds.), The Handbook of Discourse Analysis, 352-371. https://bit.ly/2MsqfRQ

Villafañe Gallego, J. (2008). Imagen positiva. Gestión estratégica de la imagen de las empresas. Pirámide.

Yu, Q., Yen, D., Barnes, B., \& Huang, Y. A. (2019). Enhancing firm performance through internal market orientation and employee organizational commitment. International Journal of Human Resource Management, 30(6), 964-987. http://doi.org/10.1080/09585192.2017.1380059 